\title{
Corrigendum: Reporters for sensitive and quantitative measurement of auxin response
}

Che-Yang Liao, Wouter Smet, Geraldine Brunoud, Saiko Yoshida, Teva Vernoux \& Dolf Weijers

Nat. Methods 12, 207-210 (2015); published online 2 February 2015; corrected after print 24 September 2015

In the version of this article initially published, it was stated that all transgenic lines were created in the Arabidopsis Col-0 ecotype. However, they were actually generated in the Columbia-Utrecht (Col-utr) ecotype. The error has been corrected in the HTML and PDF versions of the article.

\section{Corrigendum: Bayesian statistics}

Jorge López Puga, Martin Krzywinski \& Naomi Altman

Nat. Methods 12, 377-378 (2015); published online 29 April 2015; corrected after print 24 September 2015

In the version of this article initially published, the curves (in red) showing the likelihood distribution in Figure 2 were incorrectly drawn in some panels. The error has been corrected in the HTML and PDF versions of the article.

\section{Corrigendum: Comparative visualization of genotype-phenotype relationships}

Gagarine Yaikhom, Hugh Morgan, Duncan Sneddon, Ahmad Retha, Julian Atienza-Herrero, Andrew Blake, James Brown, Armida Di Fenza, Tanja Fiegel, Neil Horner, Natalie Ring, Luis Santos, Henrik Westerberg, Steve D M Brown \& Ann-Marie Mallon Nat. Methods 12, 698-699 (2015); published online 30 July 2015; corrected after print 24 September 2015

In the version of this article initially published, a funding source was omitted. The authors acknowledge additional funding from the Medical Research Council (program MC_U142684171). The error has been corrected in the HTML and PDF versions of the article.

\section{Corrigendum: A holidic medium for Drosophila melanogaster}

Matthew D W Piper, Eric Blanc, Ricardo Leitão-Gonçalves, Mingyao Yang, Xiaoli He, Nancy J Linford, Matthew P Hoddinott, Corinna Hopfen, George A Soultoukis, Christine Niemeyer, Fiona Kerr, Scott D Pletcher, Carlos Ribeiro \& Linda Partridge Nat. Methods 11, 100-105 (2014); published online 17 November 2013; corrected after print 8 August 2014; corrected after print 19 October 2015

In the version of this article initially published, only one corresponding author was listed (M.D.W.P.). L.P. has been added as a corresponding author for this article. The relevant contact information is partridge@age.mpg.de. This information has been added to the HTML and PDF versions of the article. 